\title{
Modulation of SIRT1-Foxo1 Signaling axis by Resveratrol: Implications in Skeletal Muscle Aging and Insulin Resistance
}

\author{
Thomas K. Sin Benjamin Y. Yung Parco M. Siu \\ Department of Health Technology and Informatics, Faculty of Health and Social Sciences, \\ The Hong Kong Polytechnic University, Hung Hom, Kowloon, Hong Kong, China
}

\section{Key Words}

Aging • Foxo1 • Insulin resistance • Metabolism • Resveratrol • Skeletal muscle • SIRT1

\begin{abstract}
Aging individuals and diabetic patients often exhibit concomitant reductions of skeletal muscle mass/strength and insulin sensitivity, suggesting an intimate link between muscle aging and insulin resistance. Foxo1, a member of the FOXO transcription factor family, is an important player in insulin signaling due to its inhibitory role in glucose uptake and utilization in skeletal muscle. Phosphorylation of Foxo1 is thought to mitigate the transactivation of pyruvate dehydrogenase lipoamide kinase 4 (PDK4), which is a negative regulator of the glycolytic enzyme pyruvate dehydrogenase (PDH). In contrast, how aging would regulate acetylation/deacetylation machineries and glucose utilization in skeletal muscle through the Foxo1/PDH axis remains largely undetermined. Accumulating body of evidence have shown that resveratrol, a natural polyphenol in grapes and red wine, activates the longevity-related protein sirtuin 1 (SIRT1) and augments insulin sensitivity in addition to its well-documented effects on mitochondrial energetics. The present review summarizes the role of Foxo1/ SIRT1 in insulin signaling in skeletal muscle and proposes the insight that activation of SIRT1 deacetylase activity to deacetylate and suppress the Foxo1-induced transactivation of PDK4 may represent an anti-hyperglycemic mechanism of resveratrol in aging skeletal muscle.
\end{abstract}

Copyright $\odot 2015$ S. Karger AG, Basel

Parco M. Siu, PhD,

KARGER 125
Department of Health Technology and Informatics,

The Hong Kong Polytechnic University, Hung Hom, Kowloon, Hong Kong (China)

Tel. +868523400 8593, Fax +86 8522362 4365, E-Mail parco.siu@polyu.edu.hk 
Sin/Yung/Siu: Resveratrol, SIRT1/Foxo1 and Insulin Signaling in Aging Muscle

\section{Introduction}

Skeletal muscle is an important target for anti-aging/anti-diabetic interventions provided its role in glucose clearance upon insulin stimulation and reductions of muscle mass and insulin sensitivity are not uncommon in older individuals and diabetic patients. In addition to the induction of gene products related to hepatic gluconeogenesis, aberrant changes in expression and/or post-modification of the transcription factor Foxo1 has also been implicated in the disturbance to insulin signaling and glucose uptake and utilization in skeletal muscle. The role of Foxo1 in energy homeostasis is further complicated by its plausible effects on mitochondrial metabolism. Resveratrol is a natural polyphenol found in grapes and red wine known to activate the deacetylase activity of longevity-related protein SIRT1 to enhance mitochondrial metabolism. Although emerging evidence leans support to the notion that resveratrol may orchestrate insulin and metabolic signaling through the SIRT1/Foxo1 axis, whether SIRT1 may also deacetylate and repress the hyperglycemic effects of Foxo1 in the aged skeletal muscle would need to be further investigated. This review summarizes the role of Foxo1-SIRT1 axis in insulin signaling in skeletal muscle and proposes the insight that activation of SIRT1 deacetylase activity to deacetylate and suppress the Foxo1-induced transactivation of PDK4 may represent an anti-hyperglycemic mechanism of resveratrol in the aging skeletal muscle.

\section{Intimate link between skeletal muscle aging and insulin resistance}

Muscle aging and diabetes are not mutually exclusive. Skeletal muscle is known as an effector to mediate approximately $65 \%$ of glucose clearance in response to insulin stimulation. An earlier study has demonstrated that aging elevated circulating levels of insulin and triacylglycerol and attenuated glucose uptake and utilization in the soleus muscle of obese rats [1], suggesting a relationship that exists between aging and reduced glucose clearance in skeletal muscle. Functional studies in humans have revealed that the handgrip strength was reduced with concomitant increase in homeostasis model assessment (HOMA) insulin resistance index in older, non-diabetic adults [2], thus implying that the aging skeletal muscle may have a pathogenic role in insulin resistance. The HOMA index is first described as a mathematical model predictive of pancreatic $\beta$-cell function (i.e., site of insulin production) calculated from basal plasma insulin and glucose concentrations [3]. Importantly, experimental data collected from diabetic patients unraveled that insulin resistance, rather than insulin deficiency, has a predominant role in the development of diabetes [3]. These data hence raise the possibility that skeletal muscle aging may promote the pathogenesis of diabetes whereas conditions of impaired insulin sensitivity may aggravate the functional deteriorations of the aging skeletal muscle. There appears to be an intimate link between skeletal muscle aging and insulin resistance.

\section{The need of effective anti-aging/anti-hyperglycemic regimens in the skeletal muscle}

A large amount of resources are dedicated to medical care associated with muscle aging and insulin resistance. The facts that the prevalence of diabetes increases with age and younger adults account largely for the "pre-diabetic" population exhibiting impaired glucose tolerance are worrisome [4]. According to the Centers for Disease Control and Prevention, it is estimated that older individuals would represent approximately $19.6 \%$ of the population in the United States by the year 2030 whereas an economic burden of US $\$ 218$ million was imposed by medical care and loss of productivity related to diabetes in 2007, thereby translating into an annual cost of $\$ 700$ per person for medical expenses related to diabetes [5]. Provided that diabetic patients manifest reduced muscle mass 
and strength measured as appendicular arm lean mass and grip strength, respectively [6] whereas a 30-day supplementation trial with the natural polyphenol resveratrol improves the HOMA resistance index and efficiency of energy production machinery in vastus lateralis muscle of obese men [7], it is conceivable to speculate that regimens which augment skeletal muscle mass and function in the senior population would be an attractive candidate in the prevention and treatment of diabetes.

Resistance exercise has been shown to augment muscle mass and strength in aged women [8] and increase glucose disposal in diabetic patients [9]. These beneficial outcomes, unfortunately, are often limited by the lack of motivation to exercise in the elderly population [10]. It is also noteworthy that physical inactivity is an important factor contributing to reduced muscle mass and increased prevalence of disability in older adults [11]. Nevertheless, over $50 \%$ of diabetic patients regardless of presentation of obesity have sedentary lifestyles and the likelihood to exercise regularly is reduced with increasing body mass index in nondiabetic individuals [12]. Caloric restriction, a dietary regimen involving $\sim 30 \%$ to $40 \%$ reduction of daily energy intake, has been shown to improve insulin sensitivity in both aged rats [13] and overweight, non-diabetic adults [14] but the issue of compliance in humans remains to be studied. Taken together, anti-aging/anti-diabetic interventions which gain high compliance (i.e., dietary supplementation) while mimicking the anti-hyperglycemic effects of physical exercise/caloric restriction (e.g., resveratrol) $[15,16]$ may help pronouncedly the problems of muscle aging and insulin resistance.

While the molecular mechanisms of insulin signaling cascade and their perturbations leading to insulin resistant state have been reviewed extensively elsewhere $[17,18]$, the present review attempts to discuss the perspective that modulation of the SIRT1/Foxo1related insulin signaling axis in the aging skeletal muscle might represent a novel antihyperglycemic mechanism of resveratrol.

\section{Complexity of Foxo1 signaling: crosstalk between insulin and mitochondrial signaling}

Foxo1 is a member of the "Forkhead box" (FOXO) transcription factor family which has multiple regulatory roles in signaling pathways including, but not limited to oxidative stress, apoptotic cell death, catabolism and insulin sensitivity. The physiological effects of Foxo1 originate from the binding of insulin, a hormonal peptide which promotes the reduction of circulating glucose, to its native insulin receptor (Fig. 1). This binding triggers the phosphorylation and activation of insulin receptor. The intrinsic tyrosine kinase activity of insulin receptor then phosphorylates insulin receptor substrate (IRS) to induce the canonical PI3K-Akt phosphorylation cascade (reviewed by [18-21]). This pathway involves the activation of the 2 isoforms of 3-phosphoinositide dependent protein kinase (PDK), namely PDK1 and PDK2 to phosphorylate and activate protein kinase B (Akt) at threonine 308 and serine 473, respectively. In addition, it is worth-noting that Ser473 of Akt can also be phosphorylated by mammalian target of rapamycin complex 2 (mTORC2) [22]. Recent efforts have exhibited that phosphorylation of mTORC2 at serine 2481 is essential to the induction of phospho-Akt ${ }^{\mathrm{Ser} 473}$ by insulin, an effect which was abolished by inhibition of PI3K [23]. These studies thereby suggest that Akt can be activated through multiple pathways. Activation of Akt then abrogates the gene transactivation capacity of Foxo1 through the induction of inhibitory phosphorylation at threonine 24 , serine 256 or serine 319 , leading to the retention and degradation of Foxo1 in the cytoplasm (Fig. 1).

There are studies proposing that activation of Foxo1 may have protective effects under hyperglycemic conditions. Targeted disruption of Foxo1 in liver was found to aggravate lipid abnormalities measured as triglyceride and cholesterol and up-regulate genes related to cholesterol synthesis in streptozotocin-induced diabetic mice [24]. Although a 4-hour transient exposure of hyperglycemia/hyperinsulinemia reduced the transcript levels of both isoforms of IRS, these reductions were associated with up-regulation of Foxo1 and CD36, 


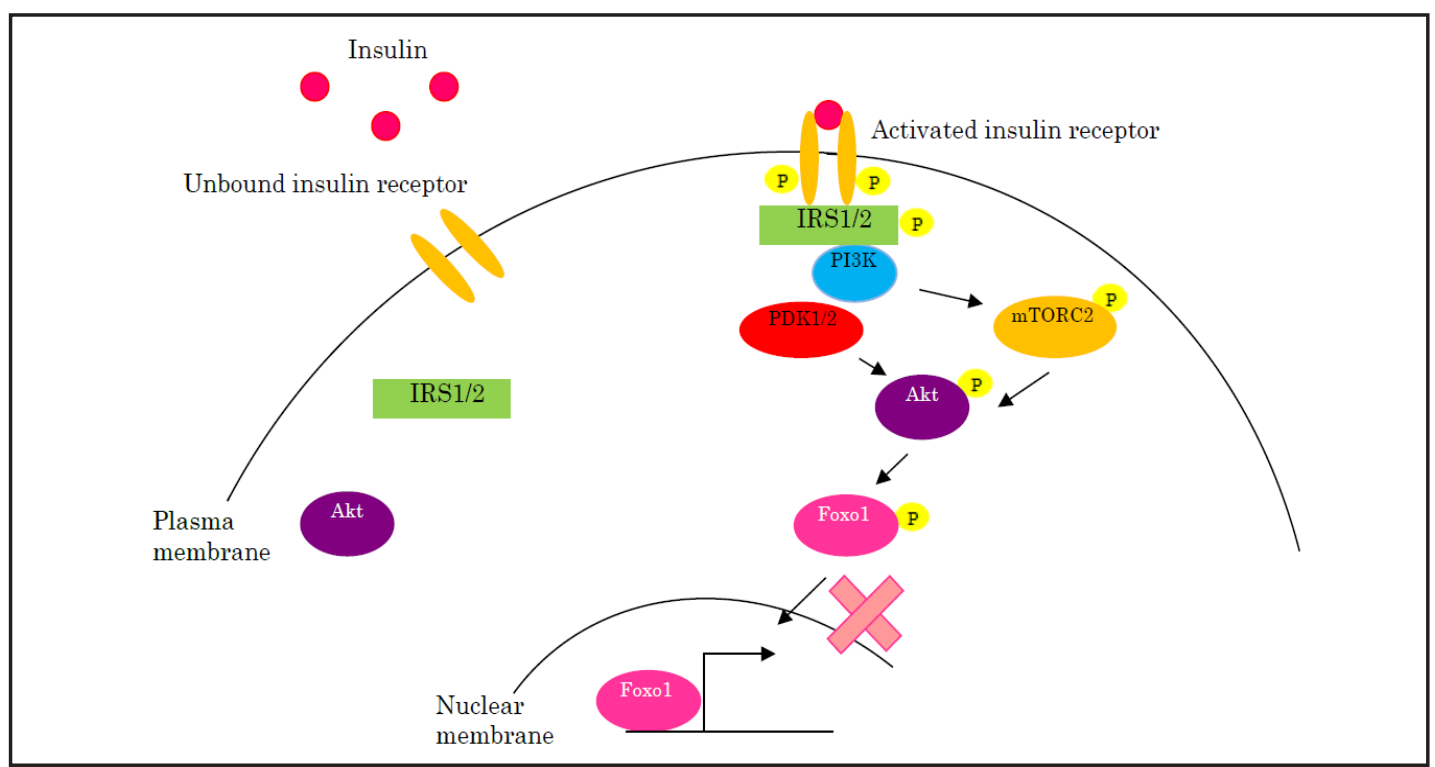

Fig. 1. The canonical insulin/Akt/Foxo1 signaling pathway. Binding of the anti-hyperglycemic hormonal peptide insulin to insulin receptor triggers a series of phosphorylation events in the chronological order of IRS, PI3K, Akt and Foxo1. It is worth-noting that Akt can be activated through multiple pathways. The phosphorylated Foxo1 can no longer translocate to the nucleus to induce transcriptional activation.

which is a gene involved in fatty acid oxidation in vastus lateralis muscle of healthy, nonobese humans [25]. These studies appear to suggest that Foxo1 may modulate mitochondrial signaling to maintain lipid homeostasis during conditions of insulin resistance. In contrast, an earlier study has demonstrated that prolonged glucose infusion for 5 days blunted the expression and membranous translocation of glucose transporter 4 (GLUT4) in the hind limb muscle of rats [26], hence raises the concern that whether insulin resistance affects pathways of mitochondrial metabolism or mitigates glucose uptake in the skeletal muscle can be largely determined and complicated by the duration of glucose infusion.

Alterations of Foxo1 have been implicated in mitochondrial adaptation associated with aging, insulin resistance and physical exercise. Previous studies have demonstrated that diabetic patients had reduced citrate synthase activity and smaller mitochondria in the vastus lateralis muscle [27] whereas senior, non-diabetic subjects exhibited poor performance in oral glucose tolerance test and reduced whole-body mitochondrial oxidative capacity [28]. Moreover, it has been reported that the mitochondrial function measured as oxygen consumption and adenosine triphosphate (ATP) production was reduced with concomitant mitigations in the protein abundances of Foxo1, PEPCK and G6pase in hepatocytes treated with berberine [29] whereas Foxo1 knockdown attenuated the reduction of mitochondrial DNA copy number induced by various muscle wasting stimuli including serum starvation, dexamethasone and myostatin in cultured C2C12 myotubes [30]. Recent findings have suggested that the phosphorylation of Foxo1 at serine 256 was reduced with up-regulation of peroxisome proliferator-activated receptor gamma coactivator 1-alpha (PGC-1 $\alpha$ ), which is the central metabolism regulator and G6pase, which is an enzyme involved in gluconeogenesis in the liver in a mouse model of hepatitis $\mathrm{C}$ infection [31]. It has also been observed in the skeletal muscle that the mRNA levels of Foxo1, pyruvate dehydrogenase lipoamide kinase (PDK4), PGC-1 $\alpha$ and aminolevulinate delta synthase 1 (ALAS1, an important protein for proper functioning of the electron transport chain) were elevated in response to acute exercise [32]. However, it remains largely unknown whether the manipulation of Foxo1 by post-translational modification machineries would affect insulin sensitivity and mitochondrial function in the aging skeletal muscle. Transactivation of Foxo1 targets in liver and skeletal muscle are illustrated in Fig. 2. 


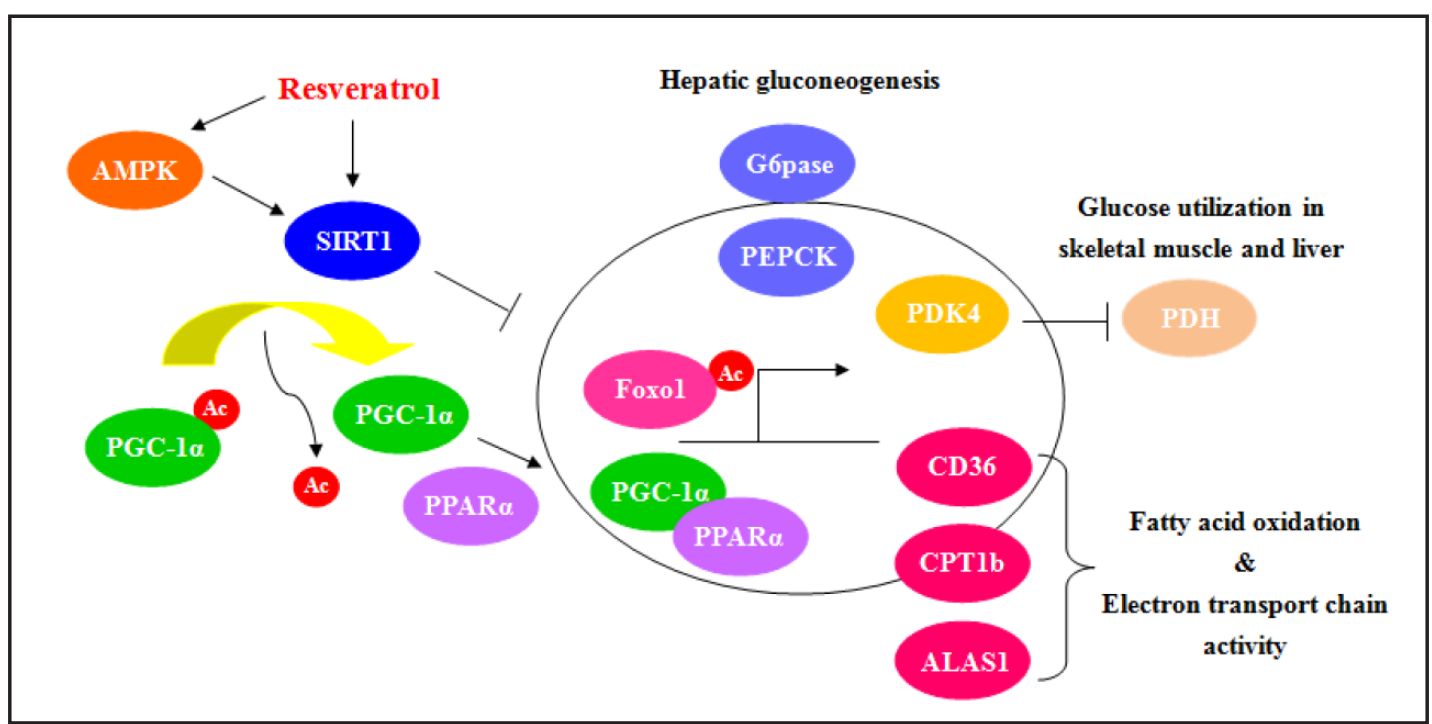

Fig. 2. Orchestration of insulin signaling and mitochondrial metabolism by Foxo 1 and PGC- $1 \alpha$. PGC-1 $\alpha$ is considered a master switch to induce transcriptional activation of genes related to mitochondrial metabolism including CD36 and CPT1b for fatty acid oxidation and functional integrity of electron transport chain including ALAS1. In addition to Foxo1, PGC-1 $\alpha$ has also been implicated in the induction of gluconeogenic genes including PEPCK and G6pase in the liver, thereby suggesting that hepatic gluconeogenesis may involve orchestration by Foxo1 and PGC-1 $\alpha$. The role of Foxo1 in insulin signaling in skeletal muscle may largely be owing to transactivation of PDK4, an inhibitor of the glycolytic enzyme PDH. More importantly, the activities of Foxo1 and PGC-1 $\alpha$ are in part governed by SIRT1-mediated deacetylation (i.e., acetylation activates Foxo1 whereas deacetylation activates PGC-1 $\alpha$ ). This raises the possibility that SIRT1 may regulate concomitantly pathways associated with insulin resistance and mitochondrial metabolism. Furthermore, the modulating effects of SIRT1 on energy homeostasis in skeletal muscle may require an upstream activating signal mediated through the energy sensor AMPK in response to resveratrol stimulation.

\section{Foxo1 as a negative regulator of glucose utilization in the skeletal muscle}

Nevertheless, an accumulating body of evidence suggests that Foxo1 is an important player during the development of insulin resistance. The concept that Foxo1 has prohyperglycemic properties is established based on a series of works that involve manipulations of the endogenous level and activity of Foxo1. Genetic ablation of Foxo1 in hypothalamus is known to enhance glucose uptake in gastrocnemius muscle [33]. Recent efforts have demonstrated that reducing the endogenous level of Foxo1 by knockdown approach abolished the elevation of MAPK phosphatase-3 (MKP-3), a factor known to induce transcriptional activation of gluconeogenic phosphoenolpyruvate carboxykinase (PEPCK) and glucose-6phosphatase complex (G6pase) in dexamethasone-treated hepatoma cells [34, 35]. Hepatic activation of mitogen-activated protein kinase (MAPK) by injection of mitogen-activated protein kinase kinase (MEK)-expressing adenovirus increased protein expression of MKP3 and fasting blood glucose despite that reduced nuclear localization of Foxo1 was noted [36]. Subsequent experiments indicated that phosphorylation-resistant Foxo1 mutants were remarkably retained in the nucleus, suggesting that post-translational modification of Foxo1 may orchestrate insulin signaling in insulin-responsive tissues [36]. In fact, it has been observed that the acetylation status of Foxo1 was reduced with concurrent transcriptional activation of glucose transporter 4 (GLUT4) and carnitine palmitoyltransferase 1b (CPT1b), an essential enzyme in fatty acid oxidation in hind limb muscles of mice subject to overnight fasting [37].

Phosphorylation of Akt/Foxo1 is considered as a molecular event that enhances insulin signaling in response to insulin stimulation, provided that the stimulus is not prolonged as KARGER 
in conditions of impaired insulin sensitivity. One of such effects are mediated by pyruvate dehydrogenase lipoamide kinase isozyme 4 (PDK4), an inhibitor of the glycolytic enzyme pyruvate dehydrogenase (PDH) (Fig. 3). It has been reported that insulin stimulation increased the phosphorylations of Akt at Ser473/Thr308 and Foxo1 at Thr24 and reduced the mRNA content of PDK4 in the gastrocnemius muscle [38]. It is noteworthy that the elevation of Foxo1 phosphorylation was accompanied by increased whole-body glucose uptake and attenuated hepatic glucose production [38]. In contrast, experimental simulation of insulin-resistant states by infusion of intralipid/lactate and insulin in rats reduced the protein contents of phosphorylated $\mathrm{Akt}^{\mathrm{Ser} 307}$ and Foxo $1^{\mathrm{Ser} 319}$ and induced transcriptional activation of PDK4 in the gastrocnemius muscle [39]. Consistent findings have shown that acute administration of the PDH activator dichloroacetate in humans mitigated the elevations of Foxo1 and PDK4 transcripts induced by an occasional high-fat diet in quadriceps muscle and reduced the level of circulating lactate during exercise [40]. These existing data thus lean support to the notion that interventions repressing the expression/activity of Foxo1 may improve glucose utilization in the skeletal muscle and thus, may have preventive/therapeutic values against insulin resistance.

\section{Activation of SIRT1 by resveratrol: more than enhancing mitochondrial metabolism}

Resveratrol (3,5,4'-hydroxystilbene) is a natural polyphenolic compound commonly found in grapes, berries and red wine and has pleiotropic effects mediated by multiple signaling pathways. The striking finding that resveratrol extends remarkably the lifespan of yeast by $70 \%$ through the activation of silent information regulator 2 (sir2) [41] has initiated an array of studies to unravel the beneficial effects of resveratrol on general health. Sirtuin 1 (SIRT1) is known as the mammalian ortholog of sir2 and is one of the most-studied members among the seven isoforms of sirtuins. It has been shown by microarray analysis that resveratrol up-regulated genes related to mitochondrial metabolism in C2C12 myocytes and skeletal muscle of mice challenged with high-fat diet [15]. However, these transcriptional changes were abrogated by treatment with SIRT1-specific shRNA, hence suggesting an indispensable role of SIRT1 [15]. It is thought that the transcriptional effects of resveratrol require the deacetylation mechanism of SIRT1. This postulation is supported by the findings demonstrating that 1) SIRT1 deacetylated and activated the central metabolism regulator PGC-1 $\alpha$ and 2) the effects of SIRT1 activation were recapitulated by infection with acetylationdefective PGC-1 $\alpha$ mutants regardless of resveratrol treatment [15]. The mechanism by which deacetylation and activation of PGC-1 $1 \alpha$ by SIRT1 leads to transcriptional activation of genes related to mitochondrial metabolism is illustrated in Fig. 2.

Although the SIRT1/PGC-1 $\alpha$ axis is generally accepted as the central signaling dogma of resveratrol-induced augmentation of mitochondrial oxidative capacity [15, 42], a growing body of studies has reported inconsistent findings. It has been previously shown by our group and others that resveratrol improved cardiac function but without affecting the expression of PGC-1 $\alpha[16,43]$. These observations are in agreement with the findings showing that an 8 -week resveratrol intervention had no effects on the protein abundances of PGC-1 $\alpha$ and complex IV subunits in triceps muscle [44]. Intriguingly, the protein contents of PGC-1 $\alpha$ and citrate synthase activity in gastrocnemius muscle were also reported to be unchanged following 10 months of resveratrol intervention [45]. Nonetheless, the exact explanations for the unaltered mitochondrial metabolic markers are not known. There are also contradictory findings indicating that forced expression of defective SIRT1 indeed enhanced acetylation of PGC- $1 \alpha$ and expression of mitochondrial proteins in both C2C12 myotubes and triceps muscle [44]. Additional research is warranted to further understand the mechanisms of these inconsistent changes in response to resveratrol treatment. In addition, these disparities bring forward the possibility that the beneficial effects of SIRT1 activation induced by resveratrol may not be necessarily confined to the induction of mitochondrial metabolism.

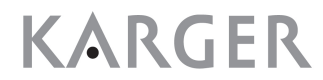




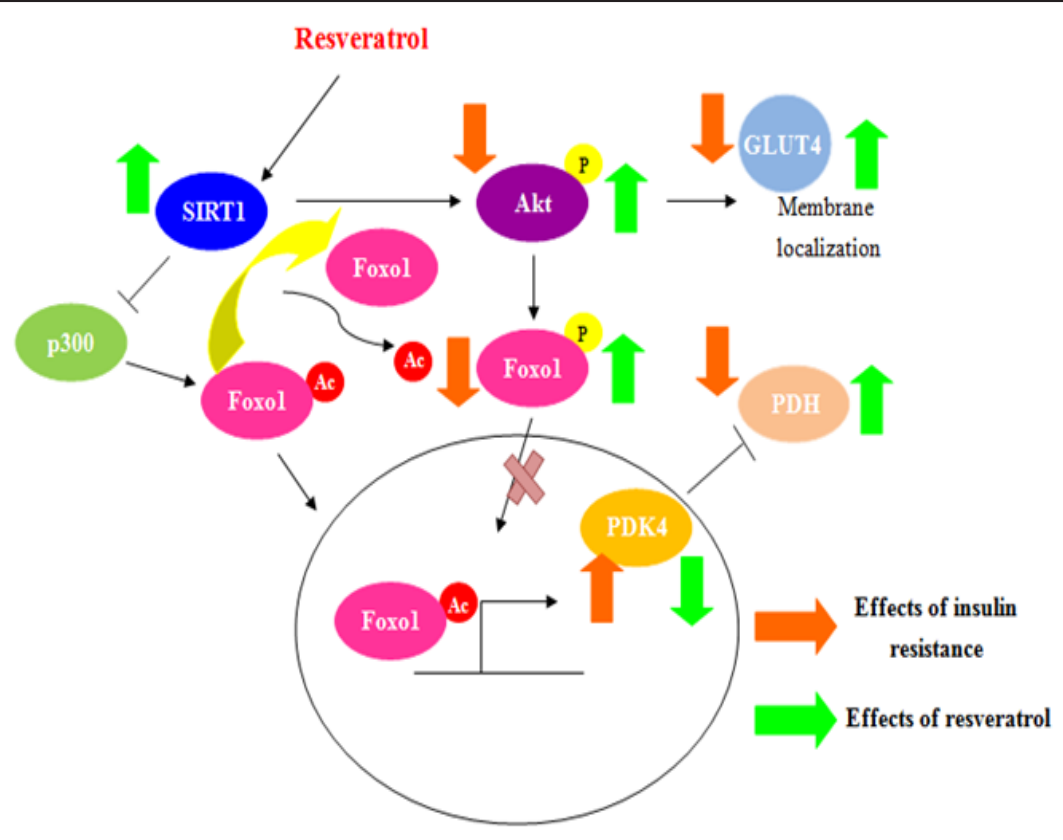

Fig. 3. Proposed mechanism of resveratrol to improve glucose clearance in the aging skeletal muscle through SIRT1/Foxo1. Foxo1 is known to induce transcriptional activation of its downstream target PDK4 to repress the activity of the glycolytic enzyme PDH. While the nuclear translocation activity of Foxo1 is thought to be inhibited upon phosphorylation, how acetylation/deacetylation machineries affect the Foxo1dependent transactivation of PDK4 remains to be determined. Resveratrol, a natural polyphenol in red wine and grapes, is a well-known activator of the longevity-related gene SIRT1. The deacetylase activity of SIRT1 may deacetylate and reduce the activity of Foxo1, resulting in increased glycolysis. In addition, it has been proposed that the deacetylation mechanism of SIRT1 is important in the phosphorylation and activation of Akt, an upstream signal which mediates the elevation of membranous GLUT4, leading to improved glucose clearance in response to insulin-stimulation.

\section{Resveratrol may confer anti-hyperglycemic effects through modulation of SIRT1-Foxo1}

It is well-documented that resveratrol enhances glucose transport/uptake in skeletal muscle. While resveratrol increases basal glucose uptake in L6 myotubes in a dose-dependent manner [46], the skeletal muscle of diabetic patients exhibits increased expression of SIRT1 and GLUT4 in response to a 12-week daily supplementation with resveratrol [47]. It has also been reported that a 16-month supplementation with resveratrol increased glucose uptake in insulin-stimulated muscles [16] and membrane localization of GLUT4 in the diabetic heart was improved in response to 5 days of resveratrol feeding [48]. However, it remains to be elucidated whether activation of SIRT1 by resveratrol may enhance the expression/ membrane localization of GLUT4, resulting in improved glucose uptake in the senescent muscle.

SIRT1 is known to modulate upstream signaling of Foxo1. Reduced contractile function of skeletal muscle has been demonstrated to accompany the down-regulation of SIRT1 and IRS1 [49]. Although further studies are required to dissect the physiological effects of SIRT1mediated deacetylation of IRS1, phosphorylation of IRS1 at serine-307 is considered as a molecular event that precedes and associates with the development of glucose intolerance $[50,51]$. The significance of Akt phosphorylation in insulin sensitivity was further confirmed in studies demonstrating that a 4-week resveratrol supplementation trial reduced postprandial glucose and increased phosphorylation of Akt at serine 473 in the platelet of diabetic patients [52] and muscle biopsies of diabetic patients manifested reduced level of KARGER 
phospho-Akt ${ }^{\text {Ser473 }}$, an effect which was mimicked by knocking down of SIRT1 in L6 myoblasts [53]. These data bring forward the idea that SIRT1 would increase phosphorylation of Akt, which is a marker indicative of intact insulin signaling. It appears that this postulation is well-supported by the observation that activation of SIRT1 deacetylase activity by caloric restriction potentiates the phosphorylation of $\mathrm{Akt}^{\mathrm{Ser} 473 / \mathrm{Thr} 308}$ induced by insulin in isolated soleus muscles [54]. Taken into consideration that the molecular mechanism by which resveratrol increases phosphorylation of Akt may at least in part, be ascribed to the exposure of Akt phosphorylation sites in response to activating deacetylation by SIRT1 [55], it is conceivable to postulate that the anti-hyperglycemic effects of resveratrol in aging muscle may require the deacetylation mechanism of SIRT1.

Most of our understandings in the role of Foxo1 in skeletal muscle physiology are acquired from experimental models of muscle disuse atrophy. These studies have shown that the capacity of Foxo1 to induce transcriptional activation of atrophic genes can be governed by acetylation/deacetylation. The histone acetyltransferase p300 has been reported to acetylate and increase nuclear localization and transactivating activity of Foxo1 in the skeletal muscle [56]. In contrast, resveratrol was observed to increase the SIRT1 deacetylase activity and alleviate the elevations of protein contents of acetylated Foxo1, atrogin1 and MuRF-1 in dexamethasone-treated L6 myotubes [57]. Although it is well-established that the activity of PDH, a glycolytic enzyme in skeletal muscle would be blunted in response to phosphorylation and inactivation of Foxo1 [38-40], how acetylation/deacetylation mechanisms would modulate the activity of Foxo1, expression of PDK4 and the subsequent glucose utilization in the aging skeletal muscle are poorly understood. It has been reported that the protein content of SIRT1 was increased with concomitant reduction of acetylation status of Foxo1 in the skeletal muscle of diabetic patients following a 3-month intake of epicatechin-containing drink [58]. Recent efforts have also unraveled that resveratrol elevated the protein contents of phospho-Foxo1 and GLUT4 in the adipose tissues of highfat diet-fed mice [59]. However, it merits further investigation whether these findings could be extrapolated in conjunction with potential involvements of acetylation/deacetylation of Foxo1 in the aging skeletal muscle. Collectively, it is plausible to speculate that activation of PDH and increased membrane trafficking of GLUT4 secondary to SIRT1-mediated deacetylation of Foxo1 may represent an anti-hyperglycemic mechanism of resveratrol (Fig. 3 ), although the validity of this speculation remains to be confirmed by future research.

\section{The SIRT1/Foxo1 axis: a pathway plausibly regulated by AMPK}

AMP-activated protein kinase (AMPK) is an energy sensor which activates in response to elevated level of adenosine monophosphate, a marker indicative of energy insufficiency. Previous works have exhibited that AMPK was required to mediate metabolic adaptation. Genetic inactivation of AMPK was observed to prevent the reduction of Foxo1 acetylation and up-regulation of GLUT4 and CPT1b in skeletal muscle of mice after 20 hours of fasting [37]. These findings are in agreement with the observation that acute bouts of swimming exercise failed to diminish the acetylation status of PGC- $1 \alpha$ in the skeletal muscle of AMPK-deficient mice [37]. It is also worth-noting that the attenuated acetylations of Foxo1 and PGC- $1 \alpha$ induced by fasting/exercise were accompanied by an increase in nicotinamide adenine dinucleotide (NAD), an effect which was abrogated in the absence of AMPK [37]. Given that NAD is a cofactor of SIRT1 deacetylase activity, the modulating effects of resveratrol/SIRT1 on energy homeostasis in skeletal muscle may require activating signal from AMPK. This speculation is supported by a recent study showing that neither resveratrol nor over-expression of SIRT1 enhanced the contents of cellular ATP and mitochondrial DNA in C2C12 cultures treated with AMPK-shRNA, an observation mimicked by pharmacological blockade of SIRT1 with EX527, implying that AMPK may act upstream of SIRT1 [60]. While the mechanisms underlying the finding that a 33-month resveratrol supplementation reduces the HOMA insulin resistance index without affecting body mass remains unclear [61], activation of AMPK by resveratrol 
may increase the enzymatic activity of SIRT1 to deacetylate and repress the hyperglycemic effects of Foxo1 and enhance the PGC- $1 \alpha$-related transcription cascade leading to improved mitochondrial metabolism (Fig. 2 \& 3). However, it has also been reported that acute exposure to higher concentrations of resveratrol mitigated phosphorylations of AMPK and Akt and glucose uptake in insulin-stimulated human myotubes [62]. Collectively, it merits further research to identify the molecular mechanisms accounting for these doserelated discrepancies and plausible cross-talk between PGC-1 $\alpha$ and Foxo1 in the context of orchestrating insulin signalling and mitochondrial metabolism in the skeletal muscle.

\section{Conclusions}

Increasing studies have demonstrated that suppression of Foxo1 at both expression and activity levels enhances insulin sensitivity, an effect largely attributable to increased glucose uptake and utilization in the skeletal muscle and reduced gluconeogenesis in the liver. In addition to the enhancement of mitochondrial metabolism through the SIRT1-PGC$1 \alpha$ axis, it is worth-noting that resveratrol, a natural polyphenol in grapes and red wine, also has anti-hyperglycemic effects including stimulation of glucose uptake and prevention of adverse alterations in insulin signaling in skeletal muscle. Despite the fact that more intense research is required before the clinical application of resveratrol as an anti-diabetic intervention, repression of the Foxo1-induced reduction in glucose uptake and utilization by activation of SIRT1 deacetylase may represent a novel, yet promising anti-hyperglycemic mechanism of resveratrol.

\section{Abbreviations}

Akt (Protein kinase B); ALAS1 (Aminolevulinate delta synthase); AMPK (AMP-activated protein kinase); ATP (Adenosine triphosphate); CD36 (Cluster of differentiation 36);CPT1b (Carnitine palmitoyltransferase 1b); Foxo1 (Forkhead transcription factor 1); G6pase (Glucose-6-phosphatase complex); GLUT4 (Glucose transporter 4); HOMA (Homeostasis model assessment); MAPK (Mitogen-activated protein kinase); MEK (Mitogen-activated protein kinase kinase); MKP-3 (MAPK phosphatase-3); mTORC2 (Mammalian target of rapamycin complex 2 (mTORC2)); MURF-1 (Muscle ring finger-1); NAD (Nicotinamide adenine dinucleotide); PDH (Pyruvate dehydrogenase); PDK1/2 (3-phosphoinositide dependent protein kinase 1/2 (PDK)); PDK4 (Pyruvate dehydrogenase lipoamide kinase isozyme 4); PEPCK (Phosphoenolpyruvate carboxykinase); PGC-1 $\alpha$ (Peroxisome proliferatoractivated receptor gamma coactivator 1-alpha); sir2 (Silent information regulator 2); SIRT1 (Sirtuin 1).

\section{Disclosure Statement}

During the writing of this article, the research activities of T.K.S. and P.M.S were supported by the Hong Kong Polytechnic University (RPTL) and the General Research Fund (PolyU 5632/10M) from the Hong Kong Research Grants Council, Hong Kong SAR. The authors would like to express the sincerest apology to those researchers whose scientific contributions were not included in this article due to the presence of constraints on citations. The authors confirm that there are no conflicts of interest to declare.

\section{References}

1 Crettaz M, Prentki M, Zaninetti D, Jeanrenaud B: Insulin resistance in soleus muscle from obese zucker rats. Involvement of several defective sites. Biochem J 1980;186:525-534.

2 Abbatecola AM, Ferrucci L, Ceda G, Russo CR, Lauretani F, Bandinelli S, Barbieri M, Valenti G, Paolisso G: Insulin resistance and muscle strength in older persons. J Gerontol A Biol Sci Med Sci 2005;60:1278-1282. 
Sin/Yung/Siu: Resveratrol, SIRT1/Foxo1 and Insulin Signaling in Aging Muscle

-3 Turner RC, Holman RR, Matthews D, Hockaday TD, Peto J: Insulin deficiency and insulin resistance interaction in diabetes: Estimation of their relative contribution by feedback analysis from basal plasma insulin and glucose concentrations. Metabolism 1979;28:1086-1096.

4 Wong KC, Wang Z: Prevalence of type 2 diabetes mellitus of chinese populations in mainland china, hong kong, and taiwan. Diabetes Res Clin Pract 2006;73:126-134.

5 Dall TM, Zhang Y, Chen YJ, Quick WW, Yang WG, Fogli J: The economic burden of diabetes. Health Aff (Millwood) 2010;29:297-303.

6 Akeroyd JM, Suarez EA, Bartali B, Chiu GR, Yang M, Schwartz AV, Araujo AB: Differences in skeletal and non-skeletal factors in a diverse sample of men with and without type 2 diabetes mellitus. J Diabetes Complications 2014

7 Timmers S, Konings E, Bilet L, Houtkooper RH, van de Weijer T, Goossens GH, Hoeks J, van der Krieken S, Ryu D, Kersten S, Moonen-Kornips E, Hesselink MK, Kunz I, Schrauwen-Hinderling VB, Blaak EE, Auwerx J, Schrauwen P: Calorie restriction-like effects of 30 days of resveratrol supplementation on energy metabolism and metabolic profile in obese humans. Cell Metab 2011;14:612-622.

8 Sipila S, Suominen H: Effects of strength and endurance training on thigh and leg muscle mass and composition in elderly women. J Appl Physiol (1985) 1995;78:334-340.

9 Holten MK, Zacho M, Gaster M, Juel C, Wojtaszewski JF, Dela F: Strength training increases insulin-mediated glucose uptake, glut 4 content, and insulin signaling in skeletal muscle in patients with type 2 diabetes. Diabetes 2004;53:294-305.

10 Burton LA, Sumukadas D: Optimal management of sarcopenia. Clin Interv Aging 2010;5:217-228.

11 Doherty TJ: Invited review: Aging and sarcopenia. J Appl Physiol (1985) 2003;95:1717-1727.

-12 Morrato EH, Hill JO, Wyatt HR, Ghushchyan V, Sullivan PW: Physical activity in u.S. Adults with diabetes and at risk for developing diabetes, 2003. Diabetes Care 2007;30:203-209.

-13 Pires RC, Souza EE, Vanzela EC, Ribeiro RA, Silva-Santos JC, Carneiro EM, Boschero AC, Amaral ME: Shortterm calorie restriction improves glucose homeostasis in old rats: Involvement of ampk. Appl Physiol Nutr Metab 2014;39:895-901.

14 Larson-Meyer DE, Heilbronn LK, Redman LM, Newcomer BR, Frisard MI, Anton S, Smith SR, Alfonso A, Ravussin E: Effect of calorie restriction with or without exercise on insulin sensitivity, beta-cell function, fat cell size, and ectopic lipid in overweight subjects. Diabetes Care 2006;29:1337-1344.

15 Lagouge M, Argmann C, Gerhart-Hines Z, Meziane H, Lerin C, Daussin F, Messadeq N, Milne J, Lambert P, Elliott P, Geny B, Laakso M, Puigserver P, Auwerx J: Resveratrol improves mitochondrial function and protects against metabolic disease by activating sirt1 and pgc-1alpha. Cell 2006;127:1109-1122.

-16 Barger JL, Kayo T, Vann JM, Arias EB, Wang J, Hacker TA, Wang Y, Raederstorff D, Morrow JD, Leeuwenburgh C, Allison DB, Saupe KW, Cartee GD, Weindruch R, Prolla TA: A low dose of dietary resveratrol partially mimics caloric restriction and retards aging parameters in mice. PLoS One 2008;3:e2264.

17 Siddle K: Signalling by insulin and igf receptors: Supporting acts and new players. J Mol Endocrinol 2011;47:R1-10.

18 Hage Hassan R, Bourron 0, Hajduch E: Defect of insulin signal in peripheral tissues: Important role of ceramide. World J Diabetes 2014;5:244-257.

19 Kido Y, Nakae J, Accili D: Clinical review 125: The insulin receptor and its cellular targets. J Clin Endocrinol Metab 2001;86:972-979.

20 Copps KD, White MF: Regulation of insulin sensitivity by serine/threonine phosphorylation of insulin receptor substrate proteins irs1 and irs2. Diabetologia 2012;55:2565-2582.

21 Rozengurt E: Mechanistic target of rapamycin (mtor): A point of convergence in the action of insulin/igf-1 and g protein-coupled receptor agonists in pancreatic cancer cells. Front Physiol 2014;5:357.

22 Sarbassov DD, Guertin DA, Ali SM, Sabatini DM: Phosphorylation and regulation of akt/pkb by the rictormtor complex. Science 2005;307:1098-1101.

23 Gan X, Wang J, Su B, Wu D: Evidence for direct activation of mtorc2 kinase activity by phosphatidylinositol 3,4,5-trisphosphate. J Biol Chem 2011;286:10998-11002.

24 Haeusler RA, Han S, Accili D: Hepatic foxo1 ablation exacerbates lipid abnormalities during hyperglycemia. J Biol Chem 2010;285:26861-26868.

25 Tsintzas K, Norton L, Chokkalingam K, Nizamani N, Cooper S, Stephens F, Billeter R, Bennett A: Independent and combined effects of acute physiological hyperglycaemia and hyperinsulinaemia on metabolic gene expression in human skeletal muscle. Clin Sci (Lond) 2013;124:675-684. 
26 Houdali B, Nguyen V, Ammon HP, Haap M, Schechinger W, Machicao F, Rett K, Haring HU, Schleicher ED: Prolonged glucose infusion into conscious rats inhibits early steps in insulin signalling and induces translocation of glut4 and protein kinase c in skeletal muscle. Diabetologia 2002;45:356-368.

27 Kelley DE, He J, Menshikova EV, Ritov VB: Dysfunction of mitochondria in human skeletal muscle in type 2 diabetes. Diabetes 2002;51:2944-2950.

28 Petersen KF, Befroy D, Dufour S, Dziura J, Ariyan C, Rothman DL, DiPietro L, Cline GW, Shulman GI: Mitochondrial dysfunction in the elderly: Possible role in insulin resistance. Science 2003;300:1140-1142.

29 Xia X, Yan J, Shen Y, Tang K, Yin J, Zhang Y, Yang D, Liang H, Ye J, Weng J: Berberine improves glucose metabolism in diabetic rats by inhibition of hepatic gluconeogenesis. PLoS One 2011;6:e16556.

30 Lokireddy S, Wijesoma IW, Teng S, Bonala S, Gluckman PD, McFarlane C, Sharma M, Kambadur R: The ubiquitin ligase mul1 induces mitophagy in skeletal muscle in response to muscle-wasting stimuli. Cell Metab 2012;16:613-624.

-31 Bernsmeier C, Calabrese D, Heim MH, Duong HT: Hepatitis c virus dysregulates glucose homeostasis by a dual mechanism involving induction of pgc1alpha and dephosphorylation of foxo1. J Viral Hepat 2014;21:9-18.

-32 Eivers SS, McGivney BA, Gu J, MacHugh DE, Katz LM, Hill EW: Pgc-1alpha encoded by the ppargc1a gene regulates oxidative energy metabolism in equine skeletal muscle during exercise. Anim Genet 2012;43:153-162.

33 Kim KW, Donato J, Jr., Berglund ED, Choi YH, Kohno D, Elias CF, Depinho RA, Elmquist JK: Foxo1 in the ventromedial hypothalamus regulates energy balance. J Clin Invest 2012;122:2578-2589.

34 Wu Z, Jiao P, Huang X, Feng B, Feng Y, Yang S, Hwang P, Du J, Nie Y, Xiao G, Xu H: Mapk phosphatase-3 promotes hepatic gluconeogenesis through dephosphorylation of forkhead box o1 in mice. J Clin Invest 2010;120:3901-3911.

-35 Feng B, He Q, Xu H: Foxo1-dependent up-regulation of map kinase phosphatase 3 (mkp-3) mediates glucocorticoid-induced hepatic lipid accumulation in mice. Mol Cell Endocrinol 2014;393:46-55.

-36 Jiao P, Feng B, Li Y, He Q Xu H: Hepatic erk activity plays a role in energy metabolism. Mol Cell Endocrinol 2013;375:157-166.

37 Canto C, Jiang LQ, Deshmukh AS, Mataki C, Coste A, Lagouge M, Zierath JR, Auwerx J: Interdependence of ampk and sirt1 for metabolic adaptation to fasting and exercise in skeletal muscle. Cell Metab 2010;11:213-219.

- 38 Fukatsu Y, Noguchi T, Hosooka T, Ogura T, Kotani K, Abe T, Shibakusa T, Inoue K, Sakai M, Tobimatsu K, Inagaki K, Yoshioka T, Matsuo M, Nakae J, Matsuki Y, Hiramatsu R, Kaku K, Okamura H, Fushiki T, Kasuga M: Muscle-specific overexpression of heparin-binding epidermal growth factor-like growth factor increases peripheral glucose disposal and insulin sensitivity. Endocrinology 2009;150:2683-2691.

-39 Kim YI, Lee FN, Choi WS, Lee S, Youn JH: Insulin regulation of skeletal muscle pdk4 mrna expression is impaired in acute insulin-resistant states. Diabetes 2006;55:2311-2317.

-40 Constantin-Teodosiu D, Constantin D, Stephens F, Laithwaite D, Greenhaff PL: The role of foxo and ppar transcription factors in diet-mediated inhibition of pdc activation and carbohydrate oxidation during exercise in humans and the role of pharmacological activation of pdc in overriding these changes. Diabetes 2012;61:1017-1024.

-41 Howitz KT, Bitterman KJ, Cohen HY, Lamming DW, Lavu S, Wood JG, Zipkin RE, Chung P, Kisielewski A, Zhang LL, Scherer B, Sinclair DA: Small molecule activators of sirtuins extend saccharomyces cerevisiae lifespan. Nature 2003;425:191-196.

42 Baur JA, Pearson KJ, Price NL, Jamieson HA, Lerin C, Kalra A, Prabhu VV, Allard JS, Lopez-Lluch G, Lewis K, Pistell PJ, Poosala S, Becker KG, Boss O, Gwinn D, Wang M, Ramaswamy S, Fishbein KW, Spencer RG, Lakatta EG, Le Couteur D, Shaw RJ, Navas P, Puigserver P, Ingram DK, de Cabo R, Sinclair DA: Resveratrol improves health and survival of mice on a high-calorie diet. Nature 2006;444:337-342.

43 Sin TK, Yu AP, Yung BY, Yip SP, Chan LW, Wong CS, Ying M, Rudd JA, Siu PM: Modulating effect of sirt1 activation induced by resveratrol on foxo1-associated apoptotic signalling in senescent heart. J Physiol 2014;592:2535-2548.

-44 Higashida K, Kim SH, Jung SR, Asaka M, Holloszy JO, Han DH: Effects of resveratrol and sirt1 on pgc-1alpha activity and mitochondrial biogenesis: A reevaluation. PLoS Biol 2013;11:e1001603.

-45 Jackson JR, Ryan MJ, Alway SE: Long-term supplementation with resveratrol alleviates oxidative stress but does not attenuate sarcopenia in aged mice. J Gerontol A Biol Sci Med Sci 2011;66:751-764. 
Sin/Yung/Siu: Resveratrol, SIRT1/Foxo1 and Insulin Signaling in Aging Muscle

46 Minakawa M, Kawano A, Miura Y, Yagasaki K: Hypoglycemic effect of resveratrol in type 2 diabetic model $\mathrm{db} / \mathrm{db}$ mice and its actions in cultured 16 myotubes and rin- $5 \mathrm{f}$ pancreatic beta-cells. J Clin Biochem Nutr 2011;48:237-244.

-47 Goh KP, Lee HY, Lau DP, Supaat W, Chan YH, Koh AF: Effects of resveratrol in patients with type 2 diabetes mellitus on skeletal muscle sirt1 expression and energy expenditure. Int J Sport Nutr Exerc Metab 2014;24:2-13.

48 Huang JP, Huang SS, Deng JY, Chang CC, Day YJ, Hung LM: Insulin and resveratrol act synergistically, preventing cardiac dysfunction in diabetes, but the advantage of resveratrol in diabetics with acute heart attack is antagonized by insulin. Free Radic Biol Med 2010;49:1710-1721.

-49 Tzika AA, Constantinou C, Bandyopadhaya A, Psychogios N, Lee S, Mindrinos M, Martyn JA, Tompkins RG, Rahme LG: A small volatile bacterial molecule triggers mitochondrial dysfunction in murine skeletal muscle. PLoS One 2013;8:e74528.

50 Deng YT, Chang TW, Lee MS, Lin JK: Suppression of free fatty acid-induced insulin resistance by phytopolyphenols in c2c12 mouse skeletal muscle cells. J Agric Food Chem 2012;60:1059-1066.

-51 Qi Y, Xu Z, Zhu Q, Thomas C, Kumar R, Feng H, Dostal DE, White MF, Baker KM, Guo S: Myocardial loss of irs1 and irs2 causes heart failure and is controlled by p38alpha mapk during insulin resistance. Diabetes 2013;62:3887-3900.

52 Brasnyo P, Molnar GA, Mohas M, Marko L, Laczy B, Cseh J, Mikolas E, Szijarto IA, Merei A, Halmai R, Meszaros LG, Sumegi B, Wittmann I: Resveratrol improves insulin sensitivity, reduces oxidative stress and activates the akt pathway in type 2 diabetic patients. Br J Nutr 2011;106:383-389.

53 Frojdo S, Durand C, Molin L, Carey AL, El-Osta A, Kingwell BA, Febbraio MA, Solari F, Vidal H, Pirola L: Phosphoinositide 3-kinase as a novel functional target for the regulation of the insulin signaling pathway by sirt1. Mol Cell Endocrinol 2011;335:166-176.

-54 Schenk S, McCurdy CE, Philp A, Chen MZ, Holliday MJ, Bandyopadhyay GK, Osborn O, Baar K, Olefsky JM: Sirt1 enhances skeletal muscle insulin sensitivity in mice during caloric restriction. J Clin Invest 2011;121:4281-4288.

-55 Sundaresan NR, Pillai VB, Wolfgeher D, Samant S, Vasudevan P, Parekh V, Raghuraman H, Cunningham JM, Gupta M, Gupta MP: The deacetylase sirt1 promotes membrane localization and activation of akt and pdk1 during tumorigenesis and cardiac hypertrophy. Sci Signal 2011;4:ra46.

-56 Senf SM, Sandesara PB, Reed SA, Judge AR: P300 acetyltransferase activity differentially regulates the localization and activity of the foxo homologues in skeletal muscle. Am J Physiol Cell Physiol 2011;300:C1490-1501.

57 Alamdari N, Aversa Z, Castillero E, Gurav A, Petkova V, Tizio S, Hasselgren PO: Resveratrol prevents dexamethasone-induced expression of the muscle atrophy-related ubiquitin ligases atrogin-1 and murf1 in cultured myotubes through a sirt1-dependent mechanism. Biochem Biophys Res Commun 2012;417:528533.

-58 Ramirez-Sanchez I, Taub PR, Ciaraldi TP, Nogueira L, Coe T, Perkins G, Hogan M, Maisel AS, Henry RR, Ceballos G, Villarreal F: (-)-epicatechin rich cocoa mediated modulation of oxidative stress regulators in skeletal muscle of heart failure and type 2 diabetes patients. Int J Cardiol 2013;168:3982-3990.

-59 Oliveira Andrade JM, Paraiso AF, Garcia ZM, Ferreira AV, Sinisterra RD, Sousa FB, Guimaraes AL, de Paula AM, Campagnole-Santos MJ, dos Santos RA, Santos SH: Cross talk between angiotensin-(1-7)/mas axis and sirtuins in adipose tissue and metabolism of high-fat feed mice. Peptides 2014;55:158-165.

60 Price NL, Gomes AP, Ling AJ, Duarte FV, Martin-Montalvo A, North BJ, Agarwal B, Ye L, Ramadori G, Teodoro JS, Hubbard BP, Varela AT, Davis JG, Varamini B, Hafner A, Moaddel R, Rolo AP, Coppari R, Palmeira CM, de Cabo R, Baur JA, Sinclair DA: Sirt1 is required for ampk activation and the beneficial effects of resveratrol on mitochondrial function. Cell Metab 2012;15:675-690.

61 Marchal J, Blanc S, Epelbaum J, Aujard F, Pifferi F: Effects of chronic calorie restriction or dietary resveratrol supplementation on insulin sensitivity markers in a primate, microcebus murinus. PLoS One 2012; 7:e34289.

62 Skrobuk P, von Kraemer S, Semenova MM, Zitting A, Koistinen HA: Acute exposure to resveratrol inhibits ampk activity in human skeletal muscle cells. Diabetologia 2012;55:3051-3060. 